\title{
Desenvolvimento participativo de um exergame para a melhora da capacidade funcional em pessoas idosas
}

\author{
Osvaldo Henrique Cemin Becker*, Jorge Luiz Andrade Da Silva Júnior, \\ Ericles Andrei Bellei, Daiana Biduski, Ana Carolina Bertoletti De Marchi
}

\author{
Instituto de Ciências Exatas e Geociências; Faculdade de Educação Física e Fisioterapia \\ Universidade de Passo Fundo, Passo Fundo - RS - Brasil \\ *175963@upf.br
}

\begin{abstract}
Resumo. Pessoas idosas comumente não atingem os nivveis recomendados de exercício físico necessário para reduzir a perda da capacidade funcional. Exergames podem ser usados como recursos para gerar engajamento. No entanto, a maioria dos exergames são disponiveis comercialmente e genéricos, usualmente não projetados para o público idoso. Neste trabalho, apresenta-se o desenvolvimento participativo de um exergame que visa a melhora da capacidade funcional em idosos. Durante o processo de criação, 23 idosos participaram contribuindo com a especificação, interagindo e testando o jogo. Os resultados trazem um exergame com mecânica e elementos mais acessíveis e atrativos para esse público, com potencial para efeitos positivos em sua saúde.
\end{abstract}

\begin{abstract}
Elderly people often do not achieve the recommended levels of exercise necessary to reduce functional decline. In this regard, exergames can be used as resources to promote exercise. However, most available exergames are generic from commercial sources and are usually not designed or tailored for the elderly. In this study, we present the participatory development of an exergame that aims to improve functional capacity in the elderly. During the creation process, 23 elderly people contributed to the specification, tested and interacted with the game. The results reveal an exergame with mechanics and elements that are more accessible and attractive to this audience, with the potential for positive effects on their health.
\end{abstract}

\section{Introdução}

A perda da capacidade funcional é um processo natural do envelhecimento humano que pode se tornar agravante à saúde dos idosos por influenciar negativamente seu desempenho e autonomia nas atividades diárias da vida cotidiana [Balsalobre-Fernández et al. 2018]. A prática de exercício físico frequente e regular é uma recomendação para melhorar a capacidade funcional dos idosos. No entanto, a inatividade física é altamente prevalente entre os idosos, que podem enfrentar dificuldades em cumprir os níveis mínimos recomendados para manter os benefícios à saúde [Taylor 2014]. A busca por maneiras adequadas e atraentes de envolver os idosos na prática de exercícios físicos é um processo constante e desafiador [Taylor et al. 2018].

Os exergames tem potencial enquanto recursos seguros e benéficos para promover a prática de exercícios físicos no público idoso [Trombetta et al. 2018]. Os exergames são jogos que necessitam de movimentos corporais, que são captados por um sensor e transferidos para o personagem do jogo ou para algum sistema de interação gestual 
[Bamparopoulos et al. 2016]. No geral, os exergames usados em intervenções com participantes idosos são jogos disponíveis comercialmente que não foram elaborados e adaptados exclusivamente para idosos, nem baseados em protocolos terapêuticos específicos [Carras et al. 2018]. Além disso, uma abordagem genérica não é a melhor solução para envolver os idosos; assim, os exergames precisam ser personalizados para corresponder aos seus objetivos e níveis de desempenho [Skjæret et al. 2016].

Um exergame com mecânica e elementos de jogo mais acessíveis para o público idoso pode ter mais chances de elicitar maior engajamento e, consequentemente, melhores resultados de saúde. O design acessível depende de recursos que podem ajudar a identificar as especificidades dos usuários, por exemplo, ouvindo-os diretamente em um processo de desenvolvimento participativo [Quintero 2020]. Partindo dessa premissa, o objetivo deste trabalho foi a criação de um exergame para a melhora da capacidade funcional em idosos utilizando-se um processo de desenvolvimento participativo.

\section{Trabalhos relacionados}

Dentre as diversas possibilidades oferecidas pela tecnologia, jogos virtuais podem combinar atividade física, dinâmica de jogo, desafios e conquistas em um ambiente confortável, mesclando elementos do mundo real com elementos virtuais [Bellei et al. 2018; Kappen et al. 2019]. Comprovadamente, exergames podem melhorar o equilíbrio, a coordenação motora e a força muscular de pessoas idosas [Stanmore et al. 2019].

Alguns estudos na literatura destacaram a importância e o êxito por integrar participantes idosos no processo de desenvolvimento. [Brox et al. 2015] forneceram um protocolo centrado no usuário para exergames adaptados às necessidades da terceira idade. Segundo os autores, o tempo dedicado aos idosos foi um elemento essencial para estabelecer a comunicação e conquistar a confiança dos participantes. [Nawaz et al. 2014] apresentaram um conceito simplificado de exergame com foco na atividade física em geral. $\mathrm{O}$ estudo foi baseado na avaliação da experiência de 7 idosos com 3 exergames disponíveis comercialmente. Os participantes afirmaram que o design dos exergames existentes deveria ser simplificado e relacionado às atividades que podem ser associadas a vida cotidiana. Por sua vez, [Chen et al. 2018] elaboraram 5 exergames com foco no bem estar e envolveram 39 participantes idosos. De acordo com os achados, a aceitação de exergames por pessoas idosas não depende tanto da diversão ao jogar, mas da utilidade percebida para melhorar as habilidades físicas e cognitivas empregadas nele.

\section{Método}

Neste estudo, foram convidados 23 idosos (idade média 68,9 $\pm 5,3$ ) frequentadores do Clube Caixeiral Campestre, na cidade de Passo Fundo, RS, Brasil. Os critérios de inclusão consideravam participantes que relataram ter aptidão para praticar atividade física por pelo menos uma vez por semana e conhecimento prévio sobre o uso de qualquer tipo de tecnologia, como smartphones e computadores. Os procedimentos foram aprovados pelo comitê de ética da Universidade de Passo Fundo, parecer 2.784.343.

Uma equipe de desenvolvimento multidisciplinar foi formada com 3 educadores físicos, 2 desenvolvedores de software (inclui-se o $1^{\circ}$ autor) e 3 especialistas em design de interação com experiência anterior no desenvolvimento de jogos para idosos. Os especialistas em design de interação auxiliaram no envolvimento dos participantes no processo de design participativo e na especificação de aspectos da interação do jogo, 
como estética, jogabilidade, níveis, pontuação, competição e outros. Os educadores físicos exploraram as possibilidades de exercícios de treinamento, auxiliaram nas orientações de jogo e nos níveis de dificuldade de acordo com os movimentos corporais necessários para completar ações no jogo. A equipe realizou reuniões para discutir aspectos relacionados à tecnologia, design do jogo, testes com interação, objetivo do estudo e resultados esperados. Os encontros eram realizados uma vez por semana, por aproximadamente 50 minutos, no clube frequentado pelos participantes.

O estudo foi guiado por 3 aspectos principais que examinaram o design da interface, o design do jogo e a experiência do jogador [Gerling et al. 2011]. Para o design e desenvolvimento de jogo, aplicou-se um processo de co-design usando a teoria do design baseado na experiência descrito por [Askenäs et al. 2019]. Também foram seguidas as orientações formuladas por [Planinc et al. 2013], que consideram o desenvolvimento de exergames controlados por movimentos corporais determinados especialmente para jogadores idosos. As orientações incluem o uso de gestos adequados, atendendo à condição física dos participantes e evitando pequenos objetos.

Durante as reuniões, o grupo debateu ideias para discutir os tipos de jogos que os idosos gostariam de jogar. A equipe de profissionais forneceu ideias sobre a escolha do jogo. Os participantes sugeriram o boliche, devido à sua semelhança com o jogo do mundo real, ou seja, a forma de jogar já thes era familiar. Eles também afirmaram que esse jogo os lembrava da atividade que praticavam com a família e amigos (aspecto motivacional). Os educadores físicos concordaram que o boliche seria uma escolha adequada, pois é um esporte de habilidade motoras fechadas que requer o ajuste fino e o treinamento de movimentos predefinidos de ritmo próprio, bem como um alto nível de concentração e equilíbrio para derrubar os pinos [DeVan and Tanaka 2007]. Além disso, o boliche permite movimentos que correspondem às atividades físicas típicas e recomendadas para pessoas mais velhas, nas quais são necessárias flexibilidade, abduções e extensões dos membros inferiores e superiores [Li et al. 2018].

Ao longo das reuniões, os participantes puderam interagir com o jogo e expressar suas percepções. O jogo foi implementado para uso com os sensores de movimento Kinect V2 ou Orbbec Astra Pro. O jogo é compatível com dispositivos com o sistema operacional Windows. Utilizou-se Unity 3D como motor de jogo, Blender para modelar e texturizar os objetos do jogo, e Adobe Fuse para a criação e modelagem dos personagens 3D. Os dados e o histórico de cada jogador são armazenados num banco de dados SQLite.

\section{Resultados}

O exergame desenvolvido foi nomeado de Boliche Virtual e teve certificado de registro de software concedido pelo Instituto Nacional da Propriedade Industrial (INPI) no processo BR512019001912-1. Há o modo de jogo individual (single player) ou em grupos (multiplayer). Foram criados 4 avatares para cada sexo, à escolha do participante. O funcionamento é similar ao jogo de boliche de 10 pinos convencional, que consiste em 10 rodadas. Em cada rodada, o jogador tem 2 chances de derrubar o máximo de pinos possível com a bola. Ao jogar no modo multiplayer, os jogadores são intercalados em cada rodada. Cada pino derrubado é igual a 1 ponto; portanto, a pontuação máxima que um jogador pode obter é 200 pontos por sessão. A sequência do jogo e o sistema de pontuação não são totalmente semelhantes ao sistema de boliche real, pois foram simplificados para facilitar a compreensão e a jogabilidade de participantes idosos. 
A Figura 1(a) exibe a seleção do jogador para visualizar o histórico de partidas. O menu tem a opção de ver o histórico de cada jogador. O lado esquerdo da tela mostra uma lista dos jogadores disponíveis. O lado direito mostra o nome do jogador com uma pontuação por partida e os resultados das partidas disputadas, ordenados pela data da partida. Existe outro botão para retornar à tela de seleção de modo na parte inferior da seleção do jogador no lado esquerdo. A principal atividade do jogo é demonstrada na Figura 1(b). O painel esquerdo exibe o nome do jogador atual e qual é o lance atual. Nesta tela, o jogador está pegando a bola para realizar sua jogada. $\mathrm{O}$ canto inferior direito mostra os movimentos do jogador conforme capturados pelo sensor de movimento. $\mathrm{O}$ botão no canto superior direito pode encerrar a partida e retornar à tela principal. A Figura 1(c) demonstra a bola rolando em direção aos pinos durante um lançamento e uma barra de progresso azul mostrando a força da bola que o jogador aplicou durante o movimento. $\mathrm{Na}$ Figura 1(d), o jogo mostra o vencedor da partida em um pódio comemorando a vitória. Duas caixas de informações mostram a pontuação de cada rodada do jogador, pontuação total, o número de strikes e spares, percentual de precisão e desempenho geral do jogador.

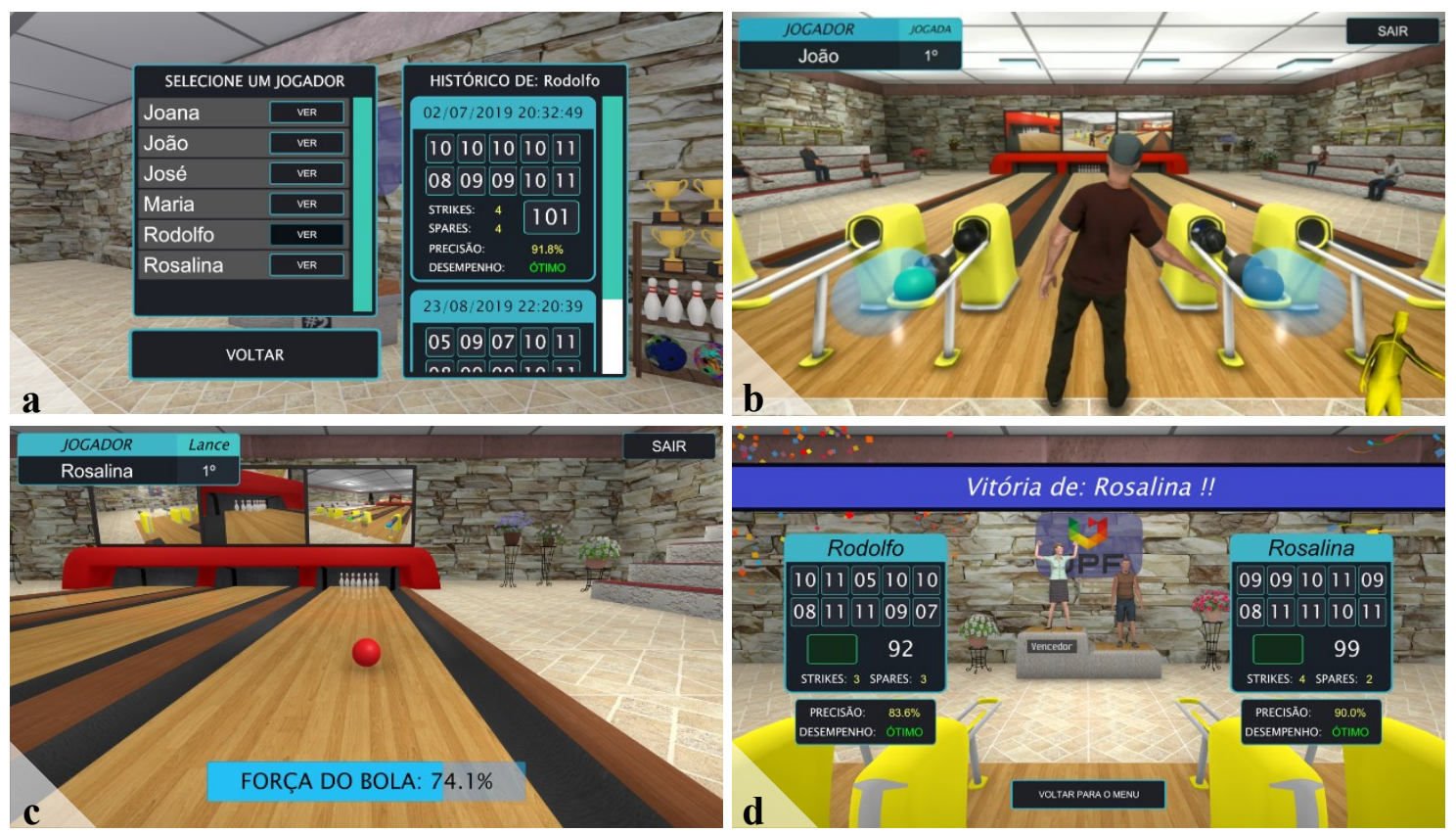

Figura 1. Capturas das principais telas do exergame Boliche Virtual.

\section{Discussão}

Compreender as oportunidades, desafios e processos de treinamento que otimizam os benefícios dos exergames tem implicações importantes para melhorar a qualidade de vida e a independência para os idosos [Zhang and Kaufman 2016]. Segundo [Meekes and Stanmore 2017], compreender a motivação de idosos para o uso de exergames pode auxiliar no processo de desenvolvimento de jogos. Foi exatamente esse benefício que este estudo buscou ao promover um desenvolvimento em que usuários são parte do processo de criação. A contribuição que o participante traz para o desenvolvimento de um produto é fundamental para o seu sucesso, pois, ao longo processo, o usuário pode ver suas sugestões sendo implementadas antes da finalização, sentindo-se valorizado e tendo mais chances de ter uma boa experiência com o produto final. [Zhang and Kaufman 2016] evidenciam que o estudo das questões de familiaridade entre os idosos é necessário para 
orientar o design de interação de novas tecnologias. Assim, eles podem ser construídos com base no conhecimento prévio de pessoas mais velhas sobre as interações do mundo real, estimulando memórias e emoções e tornando mais fácil aplicar seus conhecimentos e habilidades existentes a um novo domínio.

\section{Considerações finais}

A abordagem deste estudo ofereceu uma experiência participativa com pessoas idosas no processo de desenvolvimento de um novo exergame. Essa estratégia resultou em elementos de jogo mais atrativos e acessíveis para que os idosos possam se divertir e ter maior engajamento e motivação em fazer exercícios físicos. Consequentemente, o jogo tem potencial para elicitar desfechos positivos na saúde dos participantes. O exergame Boliche Virtual segue em desenvolvimento para adição de novos cenários, movimentos, animações e melhorias no modo multiplayer. Um artigo relatando os resultados completos e uma avaliação confirmando os efeitos benéficos na saúde de idosos foi publicado no periódico JMIR Serious Games [Da Silva Júnior et al. 2021].

\section{Agradecimentos}

Para a Fundação de Amparo à Pesquisa do Estado do Rio Grande do Sul (FAPERGS) pela bolsa de iniciação científica. Para o Conselho Nacional de Desenvolvimento Científico e Tecnológico (CNPq) pelas bolsas DTI-C, DTI-B, e DT Nível 2.

\section{Referências}

Askenäs, L., Aidemark, J., Jaarsma, T., et al. (2019). Co-design to self-organizing exergaming - a study of stimulating physical activity for elderly people with a chronic health condition. In International Conference on Internet Technologies \& Society.

Balsalobre-Fernández, C., Cordón, Á., Unquiles, N. and Muñoz-García, D. (2018). Movement velocity in the chair squat is associated with measures of functional capacity and cognition in elderly people at low risk of fall. PeerJ, v. 6, p. e4712.

Bamparopoulos, G., Konstantinidis, E., Bratsas, C. and Bamidis, P. D. (2016). Towards exergaming commons: composing the exergame ontology for publishing open game data. Journal of Biomedical Semantics, v. 7, n. 1, p. 4.

Bellei, E. A., Biduski, D., Brock, L. A., et al. (2018). Prior experience as an influencer in the Momentary User Experience : an assessment in immersive virtual reality game context. In Proceedings of 2018 20th Symposium on Virtual and Augmented Reality $(S V R)$. . IEEE Computer Society.

Brox, E., Evertsen, G., Åaheim-Olsen, H., Hors-Fraile, S. and Browne, J. (2015). Experience with a 3D Kinect Exergame for Elderly. In Proceedings of the International Conference on Health Informatics. SCITEPRESS.

Carras, M. C., Rooij, A. J. Van, Spruijt-Metz, D., et al. (2018). Commercial Video Games As Therapy: A New Research Agenda to Unlock the Potential of a Global Pastime. Frontiers in Psychiatry, v. 8.

Chen, C.-K., Tsai, T.-H., Lin, Y.-C., et al. (2018). Acceptance of different design exergames in elders. PLOS ONE, v. 13, n. 7, p. e0200185.

Da Silva Júnior, J. L. A., Biduski, D., Bellei, E. A., et al. (2021). A Bowling Exergame 
to Improve Functional Capacity in Older Adults: Co-Design, Development, and Testing to Compare the Progress of Playing Alone Versus Playing With Peers. JMIR Serious Games, v. 9, n. 1, p. e23423.

DeVan, A. E. and Tanaka, H. (2007). Declines in ten-pin bowling performance with advancing age. Age and Ageing, v. 36, n. 6, p. 693-694.

Gerling, K., Schulte, F. and Masuch, M. (2011). Designing and evaluating digital games for frail elderly persons. In ACM International Conference Proceeding Series.

Kappen, D. L., Mirza-Babaei, P. and Nacke, L. E. (2019). Older Adults' Physical Activity and Exergames: A Systematic Review. International Journal of Human-Computer Interaction, v. 35, n. 2, p. 140-167.

Li, J., Erdt, M., Chen, L., et al. (2018). The Social Effects of Exergames on Older Adults: Systematic Review and Metric Analysis. Journal of Medical Internet Research, v. 20, n. 6 , p. e10486.

Meekes, W. and Stanmore, E. K. (2017). Motivational Determinants of Exergame Participation for Older People in Assisted Living Facilities: Mixed-Methods Study. Journal of Medical Internet Research, v. 19, n. 7, p. e238.

Nawaz, A., Waerstad, M., Omholt, K., et al. (2014). Designing Simplified Exergame for Muscle and Balance Training in Seniors: A Concept of 'Out in Nature'. In Proceedings of the 8th International Conference on Pervasive Computing Technologies for Healthcare. ICST.

Planinc, R., Nake, I. and Kampel, M. (2013). Exergame Design Guidelines for Enhancing Elderly's Physical and Social Activities. In 13th International Conference on Ambient Computing, Applications, Services and Technologies.

Quintero, C. (2020). A review: accessible technology through participatory design. Disability and Rehabilitation: Assistive Technology, p. 1-7.

Skjæret, N., Nawaz, A., Morat, T., et al. (2016). Exercise and rehabilitation delivered through exergames in older adults: An integrative review of technologies, safety and efficacy. International Journal of Medical Informatics, v. 85, n. 1, p. 1-16.

Stanmore, E. K., Mavroeidi, A., De Jong, L. D., et al. (2019). The effectiveness and costeffectiveness of strength and balance Exergames to reduce falls risk for people aged 55 years and older in UK assisted living facilities: a multi-centre, cluster randomised controlled trial. BMC Medicine, v. 17, n. 1, p. 49.

Taylor, D. (2014). Physical activity is medicine for older adults. Postgraduate Medical Journal, v. 90, n. 1059, p. 26-32.

Taylor, L., Kerse, N., Klenk, J., Borotkanics, R. and Maddison, R. (2018). Exergames to Improve the Mobility of Long-Term Care Residents: A Cluster Randomized Controlled Trial. Games for Health Journal, v. 7, n. 1, p. 37-42.

Trombetta, M., Bellei, E. A., Rieder, R. and De Marchi, A. C. B. (2018). Motion Rehab 3D Plus: Um Exergame Customizável Aplicado à Reabilitação Física. In Anais do Simpósio Brasileiro de Computação Aplicada à Saúde (SBCAS). SBC.

Zhang, F. and Kaufman, D. (2016). Physical and Cognitive Impacts of Digital Games on Older Adults. Journal of Applied Gerontology, v. 35, n. 11, p. 1189-1210. 